\title{
Examining the efficacy of a novel integrative exercise-based intervention in reducing the sedentary time of a clinical population
}

\author{
Aviroop Biswas ${ }^{1,2}$, Paul I. Oh ${ }^{2}$, Guy E. Faulkner ${ }^{3}$ and David A. Alter ${ }^{1,2,4 *}$ \\ ${ }^{1}$ Institute of Health Policy, Management and Evaluation, University of Toronto, Canada \\ ${ }^{2}$ Cardiovascular Prevention and Rehabilitation Program, University Health Network-Toronto Rehabilitation Institute, Canada \\ ${ }^{3}$ School of Kinesiology, University of British Columbia, Vancouver, Canada \\ ${ }^{4}$ Institute for Clinical Evaluative Sciences, Toronto, Canada
}

\begin{abstract}
Purpose: The extent to which exercise-based interventions have spillover effects on the daily sedentary time of individuals is unclear. The objective of this study was to explore whether an integrative exercise-based intervention that had previously shown efficacy in increasing time in moderate-vigorous physical activity (MVPA) impacts the sedentary time of patients engaged in a cardiac rehabilitation (CR) program.

Methods: We examined a 12-week randomized controlled trial intervention that featured music playlists enhanced with rhythmic auditory stimulation (RAS) as compared to usual care of CR only on the duration of MVPA time and sedentary time. Accelerometer-derived and self-reported MVPA time and sedentary time were used to examine the duration spent in each. The relationship between MVPA time and sedentary time was determined using Pearson's Correlation Coefficient. Changes in MVPA time and sedentary time over the 12-weeks were determined using mixed effects repeated measures ANOVA tests.

Results: The RAS-enhanced music intervention arm showed the highest duration of weekly MVPA time across groups but irrespective of intervention exposure, all participants were highly sedentary over the 12-weeks No correlation was found between sedentary time and MVPA time of all participants derived by accelerometer $(\mathrm{r}=-0.08, \mathrm{P}=0.812)$ and self-report $(\mathrm{r}=-0.24, \mathrm{P}=<0.001)$. Self-report resulted in an overestimation of sedentary time and an underestimation of MVPA time as compared to accelerometer-derived data.

Conclusions: The majority of participants in an integrative intervention were successful at meeting MVPA recommendations but otherwise were highly sedentary. Accordingly, our findings reaffirm the needs for the need for separate strategies that focus on reducing overall daily sedentary time and promote regular MVPA.
\end{abstract}

\section{Introduction}

Prolonged sedentary time is increasingly abundant in modern societies and independently associated with cardiovascular and non-cardiovascular mortality $[1,2]$. Intervention strategies targeted at sedentary time have typically been designed to reallocate an individual's sedentary time to physical activity [3]. While evidence has established the efficacy of exercise-based interventions for increasing moderate-vigorous physical activity (MVPA), it is unclear whether these interventions have spillover effects on the daily sedentary time of individuals.

The objective of this study was to explore the extent to which an integrative exercise-based intervention impacts the sedentary time of individuals. This study utilized data obtained from a novel intervention that combined enhanced music playlists with exercise. The intervention had previously demonstrated a significant increase in participants' weekly adherence to MVPA-specific and total physical activity using music audio playlists enhanced with rhythmic auditory stimulation (RAS) as compared with a usual-care of participating in a structured exercise-based cardiac rehabilitation program. Considering its efficacy in influencing overall physical activity (irrespective of intensity), we hypothesized that the integrative exercise-based intervention effectively replaces daily sedentary time with physical activity (MVPA and otherwise).

\section{Methods}

\section{Intervention design and primary outcome findings}

The Music Activity INTervention for Adherence Improvement (MAINTAIN) study was a randomized controlled trial designed to examine the incremental effects of personalized music playlists on the time that CR patient participants spent in total and intensity-specific physical activity [4]. A total of 34 participants were initially recruited and met inclusion criteria, of which 1 participant dropped out. 33 participants completed the study; with 11 participants included in the usual-care control arm (no music intervention), and 22 participants

Correspondence to: David A. Alter, University Health Network-Toronto Rehabilitation Institute, 347 Rumsey Road, Toronto, Ontario M4G 1R7, Canada, Tel: 1-416-275-2984; Fax: 1-416-425-0301; E-mail: dalter@ices.on.ca

Key words: cardiovascular disease/prevention \& control, sedentary lifestyle, exercise, health behavior, physical fitness, risk factors

Received: June 02, 2016; Accepted: June 13, 2016; Published: June16, 2016 
received a music playlist intervention featuring two arms: (1) listening to a personalized music playlist while engaging in physical activity; (2) listening to a personalized music playlist enhanced with RAS while engaging in physical activity. All participants enrolled in the trial received $\mathrm{CR}$ as their minimum standard of care irrespective of treatment arm. Participants were recruited during their third class (3 weeks from intake) in the CR program, and were followed over the 12 -week study duration (baseline to program midpoint). The study was reviewed and approved by the University Health Network-Toronto Rehabilitation Institute research ethics board. The trial was registered with ClinicalTrials.gov I.D. (NCT01752595).

The primary outcome findings of the trial (with details on the intervention, sample size estimation and methods) are published elsewhere [4]. Briefly, the weekly average duration of total physical activity and MVPA were found to be higher among participants randomized to the RAS-enhanced music playlist intervention arm as compared to both the non-RAS music playlist arm and the nonmusic, usual-care control arm. These findings demonstrated that the integrative intervention of RAS combined with music playlists was feasible and effective at improving participation time and adherence to total and intensity-specific physical activity within a structured exercise-based cardiac rehabilitation program.

\section{Participants}

Participants were patients enrolled in the Cardiac Prevention and Rehabilitation Program of the University Health Network-Toronto Rehabilitation Institute in Toronto, Canada. The outpatient CR program is one of the largest in North America by patient volume, accommodating up to 1,800 patients a year and servicing a territory that encompasses 2.2 million mostly urban dwelling individuals. The program is publicly funded and services are provided free of charge as part of Canada's universal healthcare system. All of the patients enrolled in this study had a recent cardiovascular hospitalization. Patients were referred to the program through automated referrals at the time of hospital discharge, or by their cardiologist during ambulatory care follow-up. Waiting-time from point of referral to CR intake generally took place within 8 weeks following their cardiovascular hospitalization. Patients take part in individualized weekly exercise sessions, one-on-one counseling, education and peer-to-peer support $[5,6]$ and were expected to attend one on-site visit per week over the 24-week duration of the program.

\section{Data collection}

Patient characteristics were obtained from clinical records collected at baseline for age, sex, body mass index (by measured weight and height), cardiopulmonary fitness (peak $\mathrm{VO}_{2}$, assessed through cardiopulmonary exercise testing), referral indication, cardiovascular risk factors, comorbidities, and prior disease history. Depressive symptoms were assessed using the Centre for Epidemiological Studies - Depression Scale, and cardiac self-efficacy was assessed using the Cardiac Self Efficacy Scale [7,8].

All participants wore a triaxial accelerometer device (Personal Activity Monitor, P.A.M., model AM300, PAM BV, Doorwerth, the Netherlands) on their waist (belt, edge of pants/skirt) during their waking days to objectively measure their weekly minutes spent performing light-intensity physical activity (2 to 3 metabolic equivalents), moderate-intensity physical activity (3 to 6.9 metabolic equivalents) and vigorous-intensity physical activity ( $\geq 7$ metabolic equivalents). The P.A.M. accelerometer device is low-cost, shown to have little participant burden, capable of storing data continuously for over three months, and previously validated for the estimation of daily physical activity among adults and clinical populations $[9,10]$. Weekly recordings of moderate and vigorous intensity physical activity time were summed to estimate the MVPA for participants. MVPA time was compared to the Physical Activity Guidelines for Adults (aged 18-64 years and 65 years and older) of accumulating at least 150 minutes of MVPA per week to achieve health benefits and improve functional abilities We derived daily estimates of sedentary time using accelerometer-measured physical activity time and self-reported sleep time, which is herein referred to as the accelerometer-derived measure. While semi-objective, this composite measure of sedentary time was expected to partially correct and minimize the possible measurement errors and recall bias from using self-reported sedentary time alone $[11,12]$.

Along with accelerometer-derived data, participants were asked to self-report their daily time spent sleeping, being sedentary during their waking day, and participating in MVPA throughout the 12week duration of the MAINTAIN trial. The short-recall interval of the self-reported assessment was designed to limit the scope of allowable reporting errors. These individual participant logs were then summed into weekly totals. Weekly patient data were considered valid if nonzero values for sleeping time and sedentary time were reported for at least 5 days of the week. Excluded from calculations were sleeping and sedentary time that were reported with a zero value or left blank.

\section{Statistical analysis}

Statistical analyses were performed using the R statistical software, version 3.2 ( $\mathrm{R}$ Development Core Team, 2012) and statistical significance was established at $\mathrm{P}<0.05$. Participants in the intervention and control arms were examined separately and together for weekly differences in MVPA time and sedentary time using one-way ANOVA and Kruskal-Wallis tests. Scatterplots and Pearson product-moment correlation coefficients were examined to assess the strength and direction of the linear association between accelerometer-derived and self-reported sedentary and MVPA time of all participants. Oneway mixed effects analyses of repeated measures ANOVA tests were conducted to compare the longitudinal effect of study duration on sedentary and MVPA time. Convergent validity between the selfreported and accelerometer-derived MVPA and sedentary time were examined by Pearson correlation coefficients. Among those who provided self-reported time, one participant's accelerometer-derived data was unavailable for any of the 12 weeks and subsequently removed from the validity analysis.

\section{Results}

The baseline characteristics of all participants are detailed in Table 1. The clinical and socio-demographic characteristics of the sample was found to be generalizable to the CR patient population in the region [13]. The average age was 63 years, and the majority male, married, with at least 1-2 comorbidities and confident in their abilities to selfmanage cardiovascular disease and exercise. Poor concurrent validity was found for all participants between accelerometer-derived and self-reported sedentary time $(r=0.327, P=0.300)$ and MVPA time $(r=$ $-0.209, P=0.516$ ). Self-reported time ranged from 0 to 356 minutes of MVPA per week and 1 to 17 hours spent sedentary. Accelerometerderived MVPA ranged from 0 to 507 minutes of MVPA per week, and sedentary time ranged from 10 to 21 hours per day.

\section{Association between MVPA and sedentary time}

The RAS-enhanced music intervention arm showed the highest 
Table 1. Baseline characteristics of the study sample.

\begin{tabular}{|c|c|c|}
\hline & $\begin{array}{c}\text { Population a } \\
(\mathrm{N}=944)\end{array}$ & $\begin{array}{c}\text { Sample } \\
(n=31)\end{array}$ \\
\hline \multicolumn{3}{|l|}{ Sociodemographic factors } \\
\hline Mean age, years (SD) & $64.1(9.8)$ & $62.8(11.5)$ \\
\hline Male, n (\%) & $739(78.3)$ & $21(67.8)$ \\
\hline Married, n (\%) & $775(82.8)$ & $25(80.6)$ \\
\hline \multicolumn{3}{|l|}{ Clinical factors } \\
\hline Past or current smoker, n (\%) & $424(45)$ & $17(54.8)$ \\
\hline Mean body mass index, $\mathrm{kg} / \mathrm{m}^{2}(\mathrm{SD})$ & $29.0(5.1)$ & $28.3(3.9)$ \\
\hline Mean baseline $\mathrm{VO}_{2}$ peak, $\mathrm{ml} / \mathrm{kg} / \mathrm{min}(\mathrm{SD})$ & NA & $19.4(5.2)$ \\
\hline \multicolumn{3}{|l|}{ Referral diagnosis } \\
\hline Coronary artery bypass graft, $\mathrm{n}(\%)$ & $389(41.3)$ & $7(20.6)$ \\
\hline Percutaneous transluminal coronary angioplasty stent, $\mathrm{n}(\%)$ & $316(33.5)$ & $12(35.3)$ \\
\hline \multicolumn{3}{|l|}{ Number of comorbidities } \\
\hline None, $\mathrm{n}(\%)$ & NA & $8(23.5)$ \\
\hline $1-2, \mathrm{n}(\%)$ & NA & $18(52.9)$ \\
\hline $3-4, \mathrm{n}(\%)$ & NA & $5(14.7)$ \\
\hline \multicolumn{3}{|l|}{ Behavioral factors } \\
\hline Mean CES-D score (SD) & NA & $17.9(29.2)$ \\
\hline Mean Stanford self-efficacy score (SD) & NA & $84.5(14.8)$ \\
\hline Mean Cardiac self-efficacy score (SD) & NA & $78.7(16.3)$ \\
\hline
\end{tabular}

${ }^{a}$ Characteristics of cardiac inpatients from 11 Ontario hospitals enrolled in the Cardiac Rehabilitation Care Continuity through Automatic Referral Evaluation study (1807 of 2635 recruited patients were enrolled, 944 patients have participated in CR) (17)

$\mathrm{SD}$, standard deviation; CES-D, Centre for Epidemiological Studies - Depression scale

duration of weekly MVPA time across groups. Irrespective of intervention exposure, all participants were highly sedentary over the 12-weeks (Figure 1). We found that the association between MVPA time and sedentary time did not change between the intervention and control arms based on accelerometer-derived and self-reported data (Table 2). Among all participants, there was poor correlation between sedentary and MVPA time for both accelerometer-derived $(r=-0.08$, $P=0.812)$ and self-reported measurements $(r=-0.24, \quad P=<0.001)$. Participants underestimated their sedentary time (mean difference of 4 hours per day) and overestimated their MVPA time (mean difference of 62 minutes per week) when compared to accelerometer-derived data. Sedentary time $(\mathrm{F}$-value $=1.19, P=0.3)$ and MVPA time $(\mathrm{F}$-value $=1.623$, $P=0.093$ ) did not change significantly over the 12 -week duration of the trial.

\section{Discussion}

This study examined whether an integrative intervention combining music playlists with RAS was more efficacious at improving sedentary times than usual care among patients participating in a structured exercise-based cardiac rehabilitation program. While MVPA was increased by RAS, the integrative intervention did not influence sedentary time. This is in line with earlier work that suggests that interventions designed to increase MVPA do not elicit as meaningful of a reduction in sedentary time as those interventions focused specifically on reducing sedentary behaviors $[14,15]$.

To the best of our knowledge, this study is the first to examine the effect of an integrative intervention that combines a multicomponent lifestyle program such as CR with RAS-enhanced music playlists on displacing sedentary time. Second, we know of no other study to have examined the relationship between MVPA and sedentary time among a clinical population engaged in a structured exercise and lifestyle program. The 12-week study duration of the intervention represents the midpoint of 6-month CR and other integrative lifestyle programs, and has been shown to be a critical period in which to examine the adherence to physical activity and the uptake of healthy lifestyles [16]. The consistency in sedentary and MVPA time over this period suggests that behavioral patterns of physical activity are likely to be stable up to this point. Our finding that participants underestimated their sedentary time and overestimated their MVPA time also suggests that exercise-engaged populations seem to be no better at perceiving their activity patterns than general populations [17]. Self-reported measurement is wrought with inaccuracy related to social desirability and recall bias. While the pairing of accelerometer measurement is expected to increase precision, accuracy and to validate self-reported measures, these devices have been shown to misclassify sedentary behaviors [11]. Despite the limitations of our methods of measurement, both show consistent trends and patterns, which suggests that they are likely to realistically reflect the actual routine of patients. Future studies incorporating inclinometer devices that show promise in differentiating between postures may serve to better elucidate the risks of physical inactivity and sedentary behavior [18].

Available evidence suggests that the risks associated with prolonged sedentary time are highly dependent on MVPA. For example, a previous meta-analysis from our group suggested that adverse event rates associated with prolonged sedentary time were significantly less among those engaged in MVPA [4]. Moreover, participation in MVPA
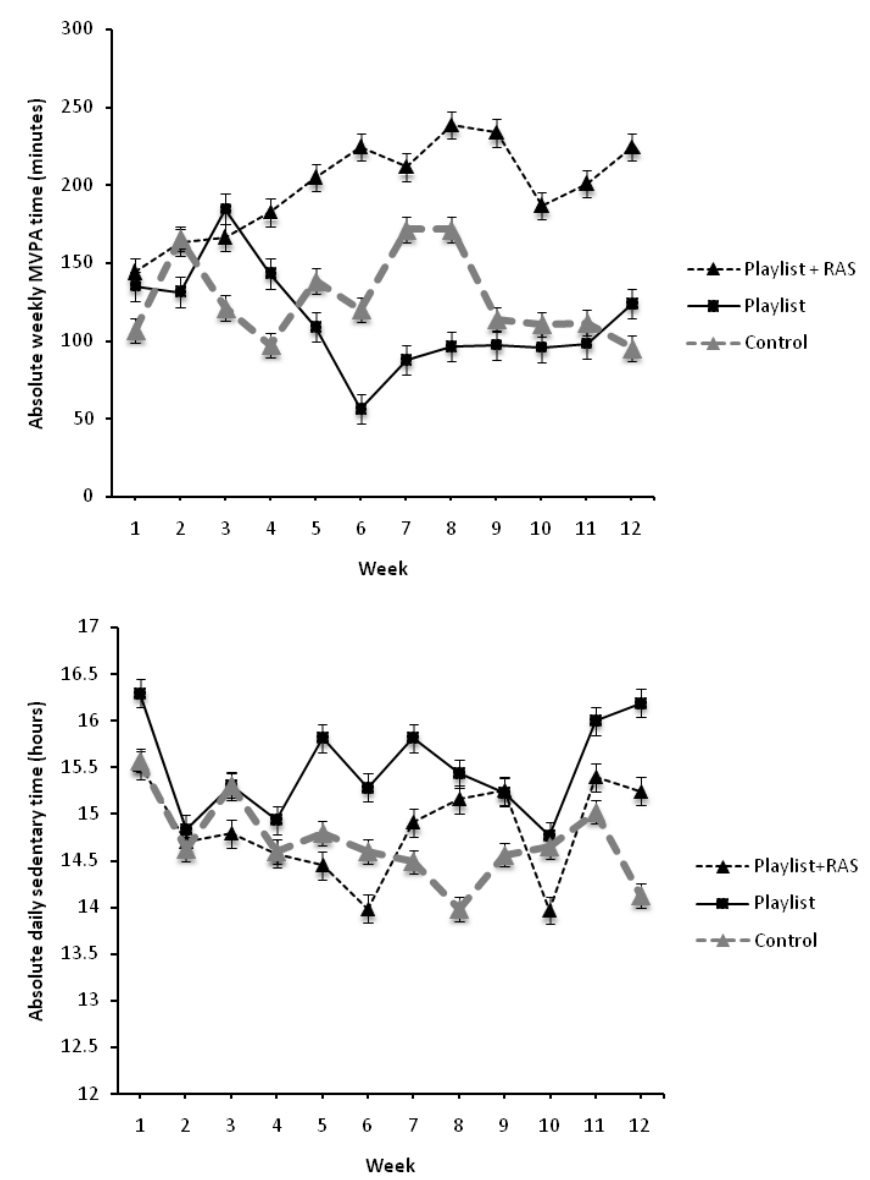

Figure 1. The moderate-vigorous intensity physical activity (MVPA)time and sedentary time of participants in an integrative intervention featuring cardiac rehabilitation (CR) with the addition of 1) music playlists enhanced with rhythmic auditory stimulation (playlist plus RAS); 2) music playlists without RAS (playlist), or 3) a control group with usual-care $\mathrm{CR}$ only. Error bars represent the standard error of the means. 
Table 2. The average weekly moderate-vigorous physical activity (MVPA) time and sedentary time of participants randomized to the trial arms of the primary randomized control trial study (Alter et al., 2015).

\begin{tabular}{|c|c|c|c|c|}
\hline & $\begin{array}{c}\text { Control arm (no music, usual } \\
\text { CR care) }(n=11)\end{array}$ & $\begin{array}{l}\text { Intervention arm (usual CR } \\
\text { care with RAS) }(n=10)\end{array}$ & $\begin{array}{c}\text { Intervention arm (usual CR } \\
\text { care without RAS) }(n=10)\end{array}$ & P value \\
\hline Weekly accelerometer-derived volume of total MVPA, mean minutes (SD) & $370.2(332.5)$ & $631.3(473.8)$ & $320(355.2)$ & $<0.001$ \\
\hline Weekly self-reported volume of total MVPA, mean minutes (SD) & $510.3(421.3)$ & $550.5(373.7)$ & $407.5(307.4)$ & $<0.001$ \\
\hline Daily accelerometer-derived volume of total sedentary time, mean hours (SD) & $13.7(2.2)$ & $14.7(1.2)$ & $13.6(2.4)$ & 0.061 \\
\hline Daily self-reported volume of total sedentary time, mean hours (SD) & $10.8(1.2)$ & $7.8(2.3)$ & $8.6(2.3)$ & 0.122 \\
\hline
\end{tabular}

SD, standard deviation; CR, cardiac rehabilitation; RAS, rhythmic auditory stimulation.

results in increasing cardiopulmonary fitness levels, which itself, is an important independent predictor of long-term outcomes over and beyond reductions in sitting times $[19,20]$. Accordingly, strategies that aim to displace sedentary time with longer durations of light-intensity physical activity may not approach the prognostic health benefits associated with shorter durations of higher-intensity physical activity, irrespective of total caloric expenditures. That said, the behavioral patterns of sedentary behavior and MVPA may be mutually exclusive for many individuals, as those who participate in MVPA may also remain sedentary during the rest of their waking day [21,22]. This may explain why studies have demonstrated only modest or negligible efficacy associated with MVPA interventions at reducing sedentary time $[15,23]$. In this study, the majority of patients who were participating in exercise-based CR and received an integrative co-intervention of personalized music playlists with RAS achieved minimal acceptable MVPA targets despite sedentary times approaching and/or exceeding 10 hours per day. As such, interventions targeting MVPA and sedentary time may necessitate separate and distinctive behavioural strategies in order for participants to sufficiently adhere to them.

This study's focus on patients participating in an integrative intervention designed to increase MVPA provides an ideal situation in which to determine the longitudinal co-occurrence of MVPA and sedentary behavior. Nonetheless, our study has limitations which merit discussion. We acknowledge that our study participants may be more compliant and adherent with prescribed physical activity than those not enrolling in such studies $[24,25]$. With this considered, the consistently long duration of sedentary time observed across trial arms and for the entire sample reinforces the expectation that there is a high burden of sedentary behavior even among exercise-engaged populations. Furthermore, a small sample size and a single center were additional limitations of this study. Accordingly, our findings are preliminary and must be further uncovered by replication among larger sample sizes.

In conclusion, the majority of participants in an integrative intervention were successful at meeting MVPA recommendations but otherwise were highly sedentary. Accordingly, while the deleterious health risks associated with prolonged sedentary time may be lower for exercise-engaged populations; the results of this study and others reaffirm the need for personalized strategies that focus on reducing overall daily sedentary time in addition to those promoting regular MVPA.

\section{Contributors}

$\mathrm{AB}$ and $\mathrm{DAA}$ wrote the manuscript and were involved in the analysis and interpretation of the data. $\mathrm{AB}$ and DAA made substantial contributions to the study conception and design. DAA, PIO and GEF revised the article for important intellectual content and approved the final version of the submitted manuscript. All authors were involved in the final approval of the manuscript. $\mathrm{AB}$ is the guarantor.

\section{Conflict of interest}

The authors have no conflicts of interest to disclose.

\section{Primary funding source}

None

\section{Financial support}

This research received no specific grant from any funding agency in the public, commercial or not-for-profit sectors. AB is supported by with an Ontario Graduate Scholarship Award. DAA is supported with a Career Investigator Award from the Heart and Stroke Foundation of Canada. GEF is supported with a Canadian Institutes of Health Research-Public Health Agency of Canada (CIHR-PHAC) Chair in Applied Public Health. PIO is supported with a GoodLife Fitness Chair in Cardiovascular Rehabilitation and Prevention, University Health Network-Toronto Rehabilitation Institute.

\section{References}

1. Colley R, Garriguet D, Janssen I, Craig C, Clarke J, et al. (2011) Physical activity of Canadian adults: accelerometer results from the 2007 to 2009 Canadian Health Measures Survey, Statistics Canada Ottawa.

2. Matthews CE, George SM, Moore SC, Bowles HR, Blair A, et al. (2012) Amount of time spent in sedentary behaviors and cause-specific mortality in US adults. Am J Clin Nutr 95: 437-445.[Crossref]

3. Owen N, Sugiyama T, Eakin EE, Gardiner PA, Tremblay MS, et al. (2011) Adults' sedentary behavior determinants and interventions. Am J Prev Med 41: 189-196. [Crossref]

4. Alter DA, O’Sullivan M, Oh PI, RedelmeierDA,Marzolini S, et al. (2015) Synchronized personalized music audio-playlists to improve adherence to physical activity among patients participating in a structured exercise program: a proof-of-principle feasibility study. Sports Med Open 1: 7.[Crossref]

5. Hamm LF, Kavanagh T (2000) The Toronto Cardiac Rehabilitation and Secondary Prevention Program: 1968 into the new millennium. J Cardiopulm Rehabil 20: 16-22. [Crossref]

6. Franklin B, Whaley M, Howley E (2013) American College of Sports Medicine guidelines for exercise testing and prescription. Williams and Wilkins, Baltimore.

7. Orme JG, Reis J, Herz EJ (1986) Factorial and discriminant validity of the Center for Epidemiological Studies Depression (CES-D) scale. J ClinPsychol 42: 28-33.[Crossref]

8. Sullivan MD, LaCroix AZ, Russo J, Katon WJ (1998) Self-efficacy and self-reported functional status in coronary heart disease: a six-month prospective study. Psychosom Med 60: 473-478. [Crossref]

9. Vooijs M, Alpay LL, Snoeck-Stroband JB, Beerthuizen T, Siemonsma PC, et al. (2014) Validity and usability of low-cost accelerometers for internet-based self-monitoring of physical activity in patients with chronic obstructive pulmonary disease. Interact J Med Res 3: e14. [Crossref]

10. Slootmaker SM, Chin A Paw MJ, Schuit AJ, van Mechelen W, Koppes LL (2009) Concurrent validity of the PAM accelerometer relative to the MTI Actigraph using oxygen consumption as a reference. Scand J Med Sci Sports 19: 36-43.[Crossref]

11. Atkin AJ, Gorely T, Clemes SA, Yates T, Edwardson C, et al. (2012) Methods of Measurement in epidemiology: sedentary Behaviour. Int J Epidemiol 41: 1460-1471. [Crossref] 
12. Matthews CE, Moore SC, George SM, Sampson J, Bowles HR (2012) Improving selfreports of active and sedentary behaviors in large epidemiologic studies. Exerc Sport Sci Rev 40: 118-126. [Crossref]

13. Shanmugasegaram S, Oh P, Reid RD, McCumber T, Grace SL (2013) Cardiac rehabilitation barriers by rurality and socioeconomic status: a cross-sectional study. Int $J$ Equity Health 12: 72.[Crossref]

14. Prince SA, Blanchard CM,GraceSL,Reid RD4 (2016) Objectively-measured sedentary time and its association with markers of cardiometabolic health and fitness among cardiac rehabilitation graduates. Eur J Prev Cardiol 23: 818-825. [Crossref]

15. Prince SA, Saunders TJ, Gresty K, Reid RD (2014) A comparison of the effectiveness of physical activity and sedentary behaviour interventions in reducing sedentary time in adults: a systematic review and meta-analysis of controlled trials. Obes Rev 15: 905919. [Crossref]

16. Lane D, Carroll D, Ring C, Beevers DG, Lip GY (2001) Predictors of attendance at cardiac rehabilitation after myocardial infarction. $J$ Psychosom Res 51: 497-501. [Crossref]

17. Klesges RC, Eck LH, Mellon MW, Fulliton W, Somes GW, et al. (1990) The accuracy of self-reports of physical activity. Med Sci Sports Exerc 22: 690-697.[Crossref]

18. Ryan CG, Grant PM, Tigbe WW, Granat MH (2006) The validity and reliability of a novel activity monitor as a measure of walking. Br J Sports Med 40: 779-784. [Crossref]
19. Warburton DE, Nicol CW, Bredin SS (2006) Health benefits of physical activity: the evidence. CMAJ 174: 801-809.[Crossref]

20. Myers J (2003) Cardiology patient pages. Exercise and cardiovascular health Circulation 107: e2-5.[Crossref]

21. Colley R, Garriguet D, Janssen I, Craig C, Clarke J, et al. (2011) Physical activity of Canadian adults: accelerometer results from the 2007 to 2009 Canadian Health Measures Survey, Statistics Canada Ottawa.

22. Owen N, Sugiyama T, Eakin EE, Gardiner PA, Tremblay MS, et al. (2011) Adults' sedentary behavior determinants and interventions. Am J Prev Med 41: 189-196. [Crossref]

23. Martin A, Fitzsimons C,JepsonR,SaundersDH,van der PloegHP,et al. (2015) Interventions with potential to reduce sedentary time in adults: systematic review and meta-analysis. Br J Sports Med 49: 1056-1063. [Crossref]

24. Alter DA, Oh PI, Chong A (2009) Relationship between cardiac rehabilitation and survival after acute cardiac hospitalization within a universal health care system. Eur J Cardiovasc Prev Rehabil 16: 102-113.[Crossref]

25. Bjarnason-Wehrens B, McGee H, Zwisler AD, Piepoli MF, Benzer W, et al. (2010) Cardiac rehabilitation in Europe: results from the European Cardiac Rehabilitation Inventory Survey. Eur J Cardiovasc Prev Rehabil 17: 410-418. [Crossref]

Copyright: (C2016 Biswas A. This is an open-access article distributed under the terms of the Creative Commons Attribution License, which permits unrestricted use, distribution, and reproduction in any medium, provided the original author and source are credited. 